\title{
Rotor Dynamic Modeling and Analysis of a Flywheel Rotor
}

\author{
${ }^{1}$ Prince Owusu-Ansah, ${ }^{1,} \mathrm{Hu}$ Yefa, ${ }^{1,}$ Adam \\ Misbawu ${ }^{2}$, \\ ${ }^{1}$ School of Mechanical and Electronic \\ Engineering, Wuhan University of Technology, \\ P.R China, 430070 \\ Kumasi Polytechnic,P.O.Box 854, Kumasi \\ Ghana- West Africa.
}

\author{
${ }^{2}$ School of Automation, Wuhan University of \\ Technology, P.R China, 430070 \\ aprinosah1990@yahoo.co.uk huyefa@163.com \\ ${ }^{2}$ misbaw6@yahoo.com
}

\begin{abstract}
This paper presents rotordynamic modeling and performance analyses of a flywheel energy storage system rotor that utilizes a hybrid magnetic bearing having an energy storage capacity of $220 \mathrm{~W}$ h at its operating speed of $20,000 \mathrm{rpm}$. A rotor model designed in solid works 13 and imported into ansys 14.5 is presented with some its main specification. Static structural analysis of the rotor has been performed using Ansys 14.5 in which the rotor model is made to undergo various analysis stages, total deformation, equivalent stress (Von- mises) and equivalent elastic strain. These results were examined and discussed, the maximum input torque for the rotor model was $4 \mathrm{NM}$ with the maximum total deformation occurring at $0.00002154 \mathrm{~mm}$ along the rotor shaft, when the rotor speed exceeds its critical speeds, influences in the behavior of the gyroscopic effects on the system results in the rotor model experiencing various degree of deformations and mechanical failures.
\end{abstract}

Key Words: Rotor dynamic, Ansys, Deformation, Critical speed, Energy storage, Mechanical failure.

\section{Introduction}

Rotor dynamics is a discipline within the study of applied mechanics which involves the vibrational behavior of axially symmetric rotating structures. A flywheel is an object that rotates at a certain velocity with a certain mass that will store energy by its speed and mass [1]. The rotor that can also be called the wheel, has been composed, in the past, by iron and other classical materials but new fibers will make the wheel less heavy and with the ability to support much higher speeds.[2].

Rotating structure are pivotal components of high speed turbo machines found in many modern day equipment's ranging from power station, automobiles, marine propulsion to high speed jet engines. These rotating structures are commonly referred to as "Rotors" [3] and generally spin about an axis at high speeds [4]. These rotors when rotates at high speed develop resonance. Resonance is the state at which harmonic loads are excited at their natural frequencies causing these rotors to vibrate excessively [5]. This vibration of larger amplitudes causes the rotors to bend and twist which leads to permanent failures. [6].

\section{Flywheel Rotor Design Principles}

Flywheels are generally used for kinetic energy storage and have been around since the early timesof man [7]. Every object rotating around an axis stores some amount of kinetic energy and could in theory be called a flywheel [8]. But the word flywheel is usually used for constructions whose main purpose is the storage of kinetic energy through rotation. This has led to the convention that the term flywheel describes a rotating, cylindrical object, usually of considerable mass, whose main purpose is to store energy or to increase the moment of inertia of a given system [9]. The total energy stored in a rotating mass can be calculated by using

$E_{k}=\frac{1}{2} I_{z} \omega^{2}$

For the sake of simplicity the flywheel is approximated to be a thin walled cylinder with radius $r$. The moment of inertia can then be calculated using $I_{z}=m_{c} r^{2}$

The total stored energy can then be expressed by substituting (2) into (1) creating the following:

$E_{k}=\frac{1}{2} m_{c} r^{2} \omega^{2}$ 
While this formula yields the total flywheel energy it is not that useful when comparing different flywheel designs. Instead, another quality is used for that purpose, namely specific energy, from here on called $Q_{k g}$. The specific energy formula is obtained by dividing (3) with the mass formula for a cylinder yielding:

$Q_{k g}=\frac{1}{2} r^{2} \omega^{2}$

However, without knowledge of the stresses in the flywheel this is not of much help. So if one instead considers a circular cross-section of a cylindrical flywheel, there are two major forces in play. One is the circumferential stress, also called hoop stress, and the other is the radial stress. In reality a third force, the axial stress, is also present. But unless the construction have some kind extra axial force, this force is usually small enough to be ignored. Approximately the hoop stress in a spinning, closed loop of wire or thin walled cylinder, with a small cross section area compared to its radius can be approximated by the following expression.

$\sigma_{\theta}=\rho \omega^{2} r^{2}$

By then combining (4) with (5) a new expression for $Q_{k g}$ can be obtained, namely:

$Q_{k g}=\frac{1}{2} \frac{\sigma_{\theta}}{\rho}$

By looking at this formula it can be concluded that, in order to build a flywheel with a high energy to weight ratio, a material with high yield strength and low density is required. Luckily there is a class of materials that exhibit such behaviors, namely lightweight composite materials. One other commonly used quality to evaluate flywheels is energy per unit volume, from here on called $Q_{\mathrm{V}}$, which is obtained by dividing (6) with the flywheel volume yielding:

$E_{k}=\frac{1}{2} \frac{m_{c} r^{2} \omega^{2}}{V} \Rightarrow \frac{1}{2} r^{2} \omega^{2} \rho$

and then combining this with (6) forming the final expression

$Q_{V}=\frac{1}{2} \sigma_{\theta}$

Figure 1. Shows a 3D model rotor designed in solidwork13 which has been imported into ansys 14.5 environment in figure 2 and the mesh model also shown in figure 3 in Ansys 14.5 environment.

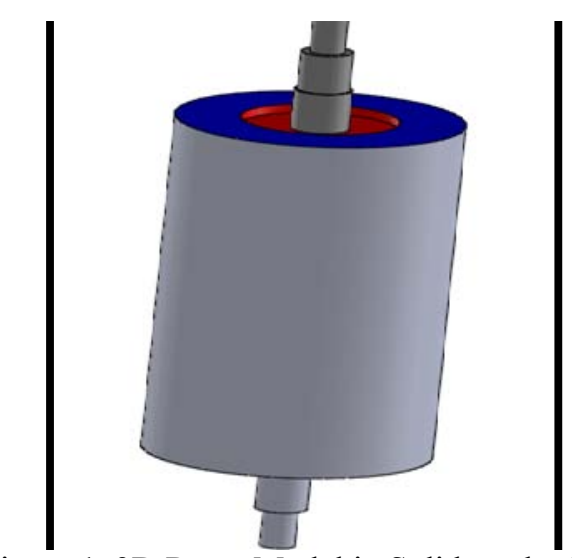

Figure 1. 3D Rotor Model in Solid works 13

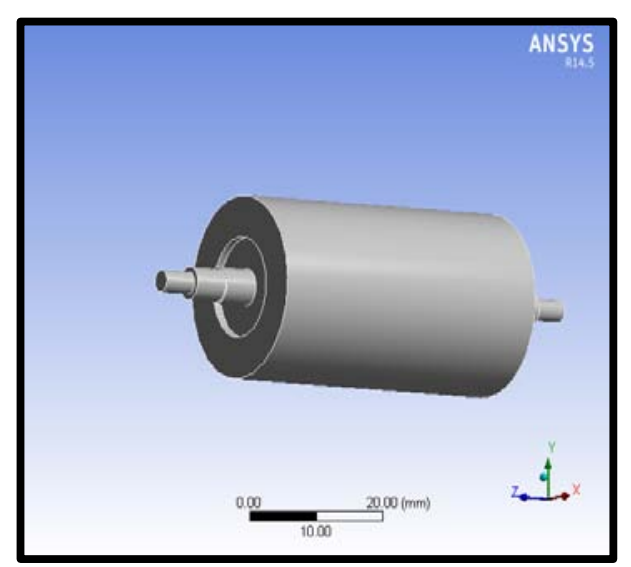

Figure 2. 3D Rotor Model in Ansys

Table 1. Shows some of the parameters of the rotor and the permanent magnetic bearing.

\begin{tabular}{ll}
\hline Name of component model & Quantity \\
\hline Rotor mass & $10 \mathrm{~kg}$ \\
Rotor diameter & $220 \mathrm{~mm}$ \\
Designed energy storage & $300 \mathrm{wh}$ \\
Maximum tip speed & $300 \mathrm{~m} / \mathrm{s}$ \\
Maximum force for upper axial AMB & $400 \mathrm{~N}$ \\
Maximum force for lower radial AMB & $550 \mathrm{~N}$ \\
Working air gap & 0.2 \\
Bias current & $1.5 \mathrm{~A}$ \\
\hline
\end{tabular}

\section{Structural Analysis}

Generally, considering the equation of multipledegrees-of-freedom systems.

$[\mathrm{M}]\{\ddot{D}\}+[\mathrm{C}]\{\dot{D}\}+[\mathrm{K}]\{\mathrm{D}\}=F$ 
Where $[\mathrm{D}]=$ displacements vector for a problem of $\mathrm{n}$ degree of freedom donated by $[\mathrm{Di}], \mathrm{i}=1,2 \ldots \mathrm{n}$.; $[\mathrm{F}]=$ external forces vector; $[\mathrm{M}]=$ mass matrix; $[\mathrm{C}]=$ damping matrix; and $[\mathrm{K}]=$ stiffness matrix.

Equation (9) represents the governing equation of a transient structural simulation. The right hand side of the equation is the external force $[\mathrm{F}]$ and the first item of the left hand side of the equation, is inertia force, is damping force, and is the elastic force.

When analyzing the free vibration of a body there is no involvement of the external force $[\mathrm{F}]$. So, equation (6), becomes

$[\mathrm{M}]\{\ddot{D}\}+[\mathrm{C}]\{\dot{D}\}+[\mathrm{K}]\{\mathrm{D}\}=0$

This finally leads to the general form of equation of motion for all vibration problems as

$[M]\{\ddot{z}\}+[C]\{\dot{z}\}+[K]\{z\}=\{f\}$

$[M]=$ symmetric mass matrix; $[C]=$ symmetric damping matrix; $[K]=$ symmetric stiffness matrix; $\{f\}=$ external force vector; $\{Z\}=$ generalized coordinate vector.

In rotor dynamics, this equation of motion can be expressed in the general form as.

$[M]\{\ddot{z}\}+([C]+[$ Cgyro $])\{\dot{z}\}+([K]+[H])\{z\}=$ $\{f\}$

Equation (15) describes the motion of an axially symmetric rotor, which is rotating at constant spin speed $(\Omega)$ about it spin axis.

The gyroscopic and circulatory matrices [Cgyro] and $[\mathrm{H}]$ are greatly influenced by rotational velocity $\Omega$, tends to zero, the skew-symmetric terms presents in equation (12) vanish and represent an ordinary stand still structure.

\section{Finite Element Analysis}

Ansys 14.5 finite element analysis software was used for the calculation of the static structure analysis and the modal analysis during the course of the rotor model analysis. To perform this process, a 3D model of the rotor was generated using solid works 2013 and subsequently imported to ANSYS 14.5 environment for the analysis to be carried out.

The material for the rotor model was chosen, the finite element mesh of the model assembly was selected, and the force, torque and the boundary conditions were defined and selected.

\section{Static Structure Analysis of the Rotor.}

After the meshing and the boundary conditions are defined and selected as shown in fig. 3, the rotor model maximum input torque was 4NM.The main objective under this section were to solve the stress, strain, and the total deformation. Total deformation: The maximum total deformation of the rotor model was $0.000021544 \mathrm{~mm}$ which is shown in figure 4 . Static structural/equivalent (Von-mises) stress: The maximum stress acting on the rotor model was $0.6812 \mathrm{Mpa}$ and the minimum stress was $0.085153 \mathrm{Mpa}$ as shown in figure 5 .

Static structural/equivalent elastic strain: Maximum elastic strain acting on the outer casing of the rotor and the shaft coupling was $3.6214 \mathrm{e}-5 \mathrm{~mm}$ and the minimum elastic strain was $1.8817 \mathrm{e}-11 \mathrm{~mm}$ as shown in figure 6. During this stage the reference position of the rotor when at rest coincides with the center of mass. This analysis offers the opportunity to be able to analysis the static structure behavior of the model to be able to understand the forms of vibrations that the model undergoes during its cycle of operational and to be to determine the maximum deformation, the equivalent stress and equivalent strain stages that the rotor model undergoes and to be able to predict the minimum and the maximum operating points of the rotor model. The maximum moment input torque was 4NM.

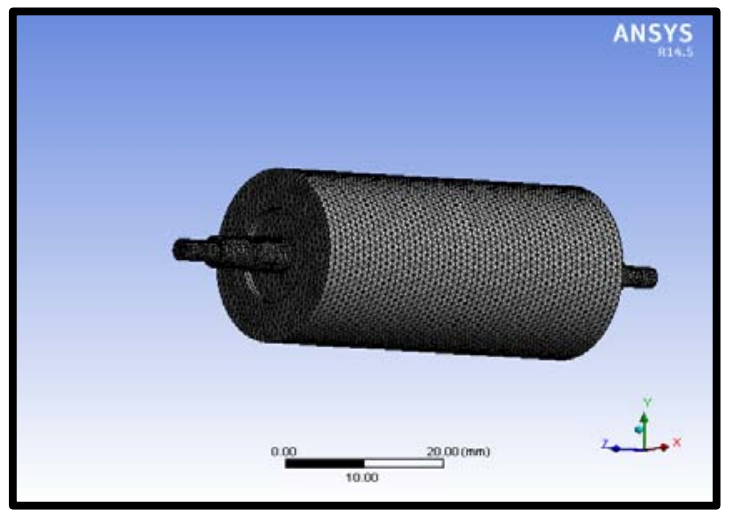

Figure 3 Meshed Rotor model in Ansys 


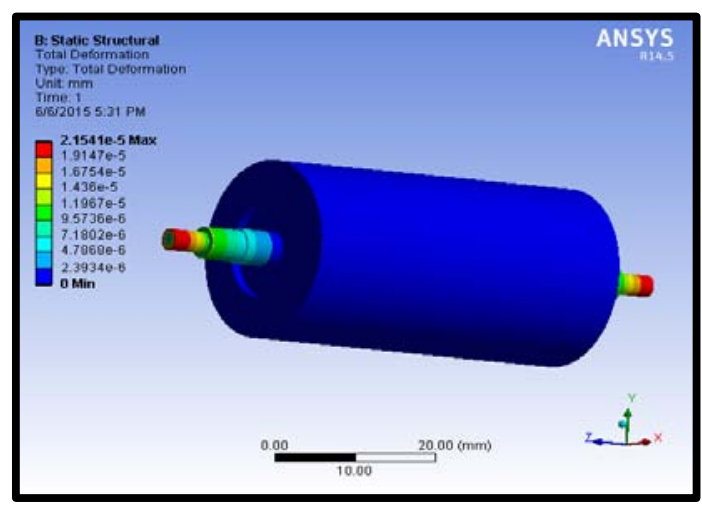

Figure 4. Total Deformation

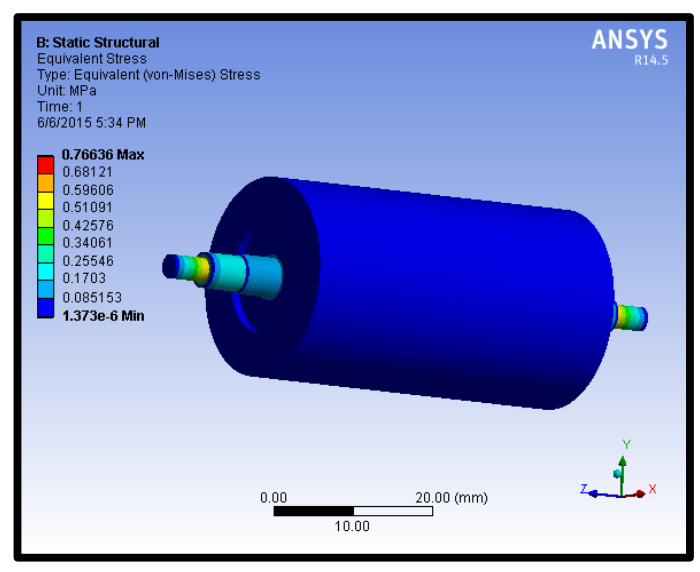

Figure 5. Equivalent Stress

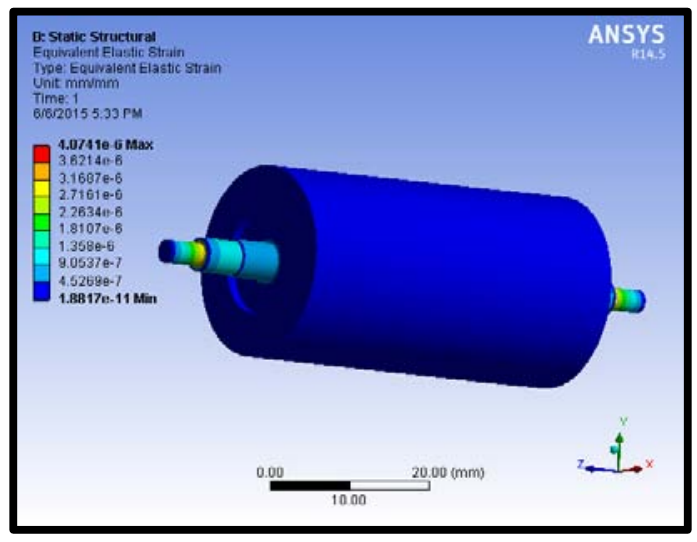

Figure 6. Equivalent Elastic Strain

\section{Conclusion}

Analyzes of total deformation, equivalent elastic strain and equivalent stress (von mises) stress of a rotor model designed in solid works 13 imported and imported into ansys 14.5 has been studied and analyzed. The results were examined and discussed, the maximum input torque for the rotor model was 4NM with the maximum total deformation occurring at $0.00002154 \mathrm{~mm}$ along the rotor shaft, the maximum stress acting on the rotor model was $0.681 \mathrm{Mpa}$ and the minimum stress were $0.085153 \mathrm{Mpa}$, the maximum elastic strain were $1.8817 \mathrm{e}-11 \mathrm{~mm}$ and occurred at the outer casing of the rotor model.

It was observed that increasing rotor rotational speed well above its safe critical limits influences the behavior of the higher gyroscopic effects on the model, as such flywheel rotors should be made to operate well below its critical speed to avoid mechanical failure and total deformation to these rotor due to the high centrifugal force which exist during high rotor speeds. Emphaises to more detailed anlysis in this area will be carried out in future works.

\section{Acknowledgment}

This work was supported by The Natural Science Foundation of China (NO.51275372) and Wuhan High-Tech Development Project Foundation (NO.201110921299) and The Fundamental Research Funds for the Central Universities (Wuhan University of Technology No.2012-IV-036)

\section{REFERENCES.}

[1] G. Genta, Kinetic energy storage Theory and practice of advanced flywheel systemsButterworth's 1985, page 58-65 ISBN 0408-01396-6.

[2] J. Vance, Fouad Zeidan, Brian Murphy, Machinery Vibration and Rotor dynamics, John Wiley \& sons, Inc., 2010.

[3] X. J. Dai., H. G.Wei and Z. P. Shen, Dynamic design and experimental study of the rotor-bearing system of a flywheel energy storage system, Chinese Journal of Mechanical Engineering, 39(4) (2003),97101.

[4] K. Zhang, L. Zhao and H. Zhao, Research on flywheel suspended by AMB's with significant gyroscope effect, Journal of Chinese Mechanical Engineering, 2004, 17(1):63-66. 
[5] M. L. Adams, Rotating Machinery Vibration: From Analysis to Troubleshooting, Marcel Dekker, Inc., 2001.

[6] B. Bolund et al, Renewable and Sustainable Energy Reviews. 11(2007): p. 235-258.

[7] S. Kumar, Rotor Dynamic Analysis using ANSYS: Proceedings of the IUTAM symposium on Emerging trends in rotor Dymanic: Edited by K. Gupta, Spring New York, 2011.

[8] W. Jeng Chen and J. Edgar Gunter, Introduction to Dynamics of Rotor-Bearing Systems, Eigen Technologies, 2001.

[9] G. Schweitzer, H. Bleuler and A. Traxle, Basic design and theory of magnetic bearings, Active Magnetic Bearings basics properties and applications, Verlagm der Fachvereine (vdf)., 1999. 